\title{
The use of platelet-rich fibrin in the surgical treatment of medication-related osteonecrosis of the jaw: 40 patients prospective study
}

\author{
Jiri Zelinka ${ }^{a}$, Jiri Blahaka, Vojtech Perina ${ }^{a}$, Rita Pacasova ${ }^{b}$, Jana Treglerovaa ${ }^{a}$ Oliver Bulik ${ }^{a}$
}

\begin{abstract}
Objectives. Medication-related osteonecrosis of the jaw (MRONJ) is defined as exposed bone in the maxillofacial region persisting for more than eight weeks in patients who are or were treated with antiresorptive or antiangiogenic agents and had no radiation therapy to the craniofacial region or obvious metastatic disease of the jaws. It is a recognised side effect of antiresorptive or antiangiogenic medication. To date, there is no specific gold standard treatment for MRONJ cases. The aim of this study was to evaluate the successful rate of surgical treatment with adjuvant local application of platelet rich fibrin.
\end{abstract}

Methods. 40 patients treated with necrotic bone resection and adjuvant local application of platelet-rich fibrin (PRF) were included. Treatment outcomes were evaluated after 12 months.

Results. The outcome of surgical treatment was successful in 34 of all 40 patients (85\%), in 12 months follow-up. If we evaluate only cases where removal of all necrotic bone was possible the success rate was increased to $94 \%$. A significant association between size of necrotic bone and treatment response was found ( $P=0.014$, Wilcoxon rank sum test with continuity correction).

Conclusions. Surgical treatment of MRONJ with adjuvant local PRF application proved to be very effective and safe, especially in early stages when all necrotic bone can be easily removed.

Key words: medication related osteonecrosis of the jaw, platelet rich fibrin, surgical treatment

Received: February 2, 2020; Revised: May 2, 2020; Accepted: May 12, 2020; Available online: June 26, 2020 https://doi.org/10.5507/bp.2020.023

() 2021 The Authors; https://creativecommons.org/licenses/by/4.0/

${ }^{a}$ Clinic of Oral and Maxillofacial Surgery, University Hospital Brno, Czech Republic

${ }^{b}$ Transfusion and Tissue Department, University Hospital Brno, Czech Republic

Corresponding author: Jiri Zelinka, e-mail:zelinka.jiri@fnbrno.cz

\section{INTRODUCTION}

Medication-related osteonecrosis of the jaws (MRONJ) was first reported by Marx in 2003. He presented a group of patients with avascular osteonecrosis of the jaws, who were treated with bisphosphonates for cancer or osteoporosis and named this disease bisphosphonate-related osteonecrosis of the jaws (BRONJ) (ref. $\left.{ }^{1}\right)$. Later it was recognized that the same necrosis of the jaws were associated with other antiresorptive and antiangiogenic agents and it was recommended to change the nomenclature from BRONJ to the less specific term medication-related osteonecrosis of the jaw. According to the American Association of Oral and Maxillofacial Surgeons (AAOMS) is MRONJ defined as exposed bone or bone that can be probed through an intraoral or extraoral fistula(e) in the maxillofacial region that has persisted for more than eight weeks in patients who are or were treated with antiresorptive or antiangiogenic agents and had no radiation therapy to the craniofacial region or obvious metastatic disease to the jaws. AAOMS also defined five stages of MRONJ (at risk and 0-3) (ref. ${ }^{2}$ ). Though it is more than 15 years since the first description of BRONJ/MRONJ and MRONJ has become an object of extensive research, the pathogenesis of MRONJ is still not completely understood and there is also still no consensus about best treatment strategy, though a conservative approach is usually recommended as the first choice. AAOMS suggests use of oral antimicrobial rinses, such as chlorhexidine $0.12 \%$, systemic antibiotic therapy, pain control and debridement to relieve soft tissue irritation for stages one and two. Surgical treatment is recommended only for the third stage ${ }^{2}$. Also in a position paper of the Allied Task Force Committee of the Japanese Society for Bone and Mineral Research, Japan Osteoporosis Society, Japanese Society of Periodontology, Japanese Society for Oral and Maxillofacial Radiology, and Japanese Society of Oral and Maxillofacial Surgeons conservative approach is recommended ${ }^{3}$. The problem with conservative treatment is low success rate. It can reduce clinical signs such as pain and inflammation but usually does not resolve exposed bone. Complete mucosal healing is achieved in about $20 \%$ cases $^{46}$. Therefore surgical therapy is gradually gaining more importance. Otto et al. proposed surgical treatment for all stages of MRONJ according to the AAOMS classification. The rationale for this approach is the higher success rate of mucosal healing, removing exposed necrotic bone and hermetic wound closure to prevent bacterial infection and progression to higher stages of MRONJ, shorter time of therapy and histopathological confirmation of diagnosis ${ }^{7}$. Various adjuvant treatment methods such as ozone, laser and hyperbaric oxygenation have been used to improve outcomes of surgical treatment ${ }^{8-10}$. In this study the suc- 
cess rate of surgical treatment with adjuvant local application of platelet-rich fibrin (PRF) was assessed. PRF was first described by Choukroun et al. specially for use in the maxillofacial region. This is an autologous platelet concentrate that contains high concentrations of various growth factors with the potential to stimulate bone regeneration, angiogenesis as well as soft tissue healing such as platelet-derived growth factor (PDGF), transforming growth factor, vascular endothelial growth factor (VEGF) and epithelial growth factor. Unlike platelet-rich plasma (PRP) PRF is prepared from blood without addition of anticoagulants or coagulation activators. It is referred to as second generation platelet concentrate. Platelet rich fibrin, compared to PRP, has some favourable properties such as slow and extended release of growth factors, more stable and coherent architecture of the fibrin matrix which serves as a scaffold for cells taking part in the healing process ${ }^{11-14}$.

\section{MATERIAL AND METHODS}

48 consecutive patients treated at the Clinic of Oral and Maxillofacial Surgery, University Hospital Brno, Czech Republic with MRONJ were involved in this single group prospective study. Inclusion criteria were: diagnosis of MRONJ of all stages according to AAOMS definition, adult patients able to sign an informed consent form. Excluded criteria: patients unable to undergo surgical treatment under local or general anaesthesia, patients who disagreed with surgical therapy, patients with contraindications for PRF use: platelet dysfunction syndrome, critical thrombocytopenia, septicaemia, presence of precancerous or malignant lesions near the location of application of PRF. Patients were examined by a maxillofacial surgeon who confirmed the diagnosis of MRONJ, described site of osteonecrosis and size of exposed bone, stage of disease according to AAOMS classification. Type of antiresorptive or antiangiogenic medication, duration of use and indication for use, triggering factors (extractions, pressure sores, periodontal infection), other risk factor (chemotherapy, diabetes, smoking, use of corticosteroids) and information about previous treatment were recorded. Pain intensity was measured with visual analogue scale 0-10 (VAS).

\section{PRF production}

L-PRF was prepared before surgery according to the Choukrouns protocol ${ }^{11}$. Patient's venous blood was taken into 5-ml glass tubes (BD Vacutainer ${ }^{\circledR}$, BD-Plymouth, UK) without anticoagulant (from 4 to 12 tubes per patient) and immediately centrifuged (EBA 20, Hettich $\mathrm{GmbH}$ and Co., Germany) at $3200 \mathrm{rpm}$ (approximately $401 \mathrm{~g}$ ) for $10 \mathrm{~min}$.

\section{Surgical treatment}

Oral antibiotic treatment with amoxicillin and clavulanic acid $(875 \mathrm{mg} / 125 \mathrm{mg}$ ) bid and in case of allergy to penicillin, clindamycin $300 \mathrm{mg}$ tid was initiated two days before surgery. Depending on the size of necrosis and patient general condition, surgery was performed under local or general anaesthesia. All sequestrectomies were performed via the intraoral approach. After preparation of the operating field and infiltration area with articain 4\% with 1:200 000 epinephrine, the mucoperiosteal flap was elevated, necrotic bone was removed with rotating burs and all sharp edges were smoothed. An attempt to remove all necrotic bone was made where possible, extent of resection was based on macroscopic appearance of bone. In four cases not all necrotic bone was resected because of the poor general health condition of the patient, or where the resection of all necrotic bone would lead to mandibular continuity defect in the chin region and need for tracheostomy. Subsequently the surgical site was meticulously irrigated, the mucoperiosteal flap was mobilised to facilitate tension-free closure and any bleeding was controlled by electrocoagulation. L-PRF was obtained from the middle of the tube, put on the surgical table, red blood clot was discarded (except a small part in contact with fibrin clot) and the volume of fibrin clot was reduced by application of light pressure with a wet gauze. Bone defect after resection was filled with PRF clots and the wound was closed in a watertight manner with resorbable 4-0 sutures. All surgical procedures were performed by one surgeon. Postoperatively, intravenous antibiotics were prescribed for one week and then oral antibiotics for another (Fig. 1-3).

\section{Follow up}

Patients were scheduled for follow-up control visits 2 weeks, one, two, three and six months and one year postoperatively.

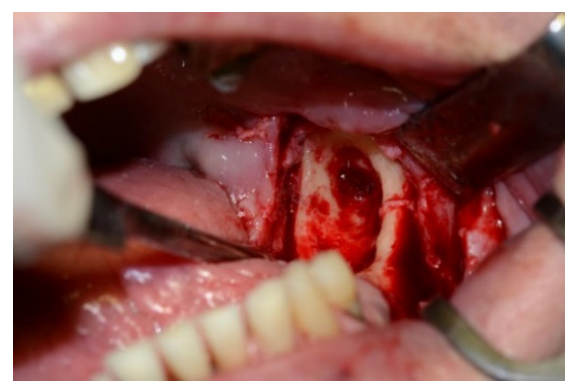

Fig. 1. Operation site after resection of necrotic bone.

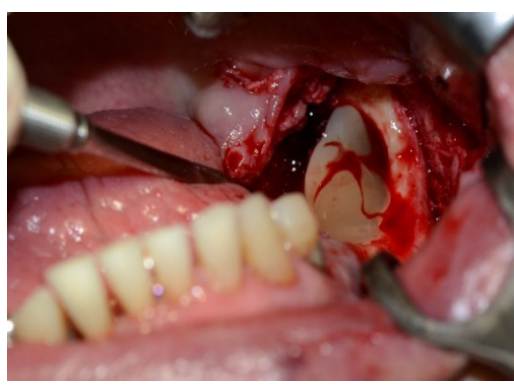

Fig. 2. Application of PRF clot to the defect.

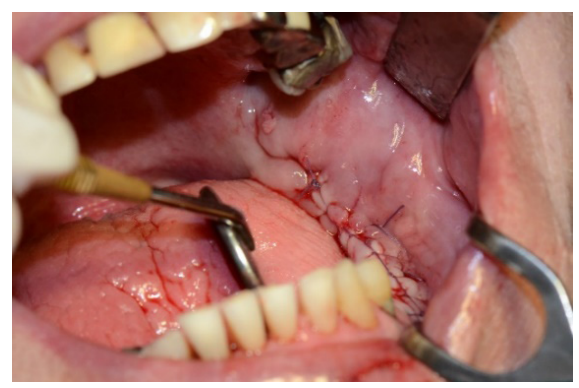

Fig. 3. Hermetic closure. 


\section{RESULTS}

48 patients underwent surgery, 8 of them were excluded from final evaluation because their follow up was shorter than 1 year. Treatment was assessed as successful in the case of no exposed bone at the site, fully covered by intact mucous membrane, with no pain or signs of inflammation. $24(60 \%)$ patients were female, $16(40 \%)$ were male, the mean age was 69 years (range $37-85$ years). 34 ( $85 \%$ ) patients had malignant disease, 6 were treated for osteoporosis. Diagnoses are presented in table 1. Twenty-five lesions (62.5\%) were located in the lower jaw, fifteen (37.5\%) in the upper jaw, stages of MRONJ according to AAOMS are presented in the Table 2.

The size of the MRONJ was approximate during the operation after clarifying borders of osteonecrosis as rectangular area.

The most common triggering event was tooth extraction. This was the cause of MRONJ in 27 cases (67.5\%), in $12(30 \%)$ cases MRONJ was triggered by pressure sores and in one case $(2.5 \%)$ by periapical infection. Ten of the patients were treated only by bisphosphonates (25\%), 13 (32.5\%) only by denosumab and 17 (42.5\%) had in their medical history both bisphosphonates and denosumab. The mean duration of medication before MRONJ occurrence for all patients was 52.8 months, median 43 months, (range from 4 to 144 months).

For high-dose antiresorptive drug treatment (oncological patients) mean duration was 51.5 months, range from 4 to 144 months and for low-dose treatment, the mean duration was 60.2 months and range from 31 to 109 months.

We recorded data on systemic therapy that is known risk factor for MRONJ development and poor healing. Eleven patients $(27.5 \%)$ had chemotherapy in the perioperative period, 9 were treated with chemotherapy and corticosteroids $(22.5 \%)$, and $2(5 \%)$ with corticosteroids, 18 patients (45\%) had none of these risk medications. Other registered risk factors were smoking (two patients, $5 \%$ ) and diabetes (twelve patients, 12.5\%), two patients were smokers with diabetes $(5 \%)$. We also collected data on previous unsuccessful treatment. Twenty-one patients were treated conservatively with antibiotics (52.5\%), 14 patients (35\%) underwent surgical therapy with antibiotics and only five patients $(12.5 \%)$ had no previous treatment.

In the presented study the outcome of surgical treatment with local PRF application was successful in 34 of the 40 patients $(85 \%)$.

Characteristics of 6 patients with recurrence of the MRONJ:

1. Female, age 69, MRONJ in lower jaw, stage 2 (purulent discharge), VAS:0, diagnosis: lung carcinoma, antiresorptive drug denosumab (Xgeva), duration of therapy 22 months, other risk factor: chemotherapy; size of mucosal dehiscence $3 \times 2 \mathrm{~mm}$, area of necrotic bone $35 \times 10 \mathrm{~mm}$, recurrence after 6 months ( 3 months after restart of Xgeva treatment), one year after treatment VAS 0 , stage 1 , size of dehiscence $3 \times 1 \mathrm{~mm}$.

2. Female, age 66, MRONJ in lower jaw, stage 2,VAS:8, diagnosis: breast cancer, antiresorptive drug deno-
Table 1. List of primary diagnoses.

\begin{tabular}{ll}
\hline Diagnosis & Counts \\
\hline Renal cancer & $2(5 \%)$ \\
Pancreatic cancer & $1(2.5 \%)$ \\
Lung cancer & $3(7.5 \%)$ \\
Prostate cancer & $14(35 \%)$ \\
Breast cancer & $12(30 \%)$ \\
Brest cancer and B lymphoma & $1(2.5 \%)$ \\
Myeloma & $1(2.5 \%)$ \\
Osteoporosis & $6(15 \%)$ \\
\hline
\end{tabular}

Table 2. Stages of MRONJ.

\begin{tabular}{cc}
\hline Stage & Counts \\
\hline 0 & $1(2.5 \%)$ \\
1 & $3(7.5 \%)$ \\
2 & $21(52.5 \%)$ \\
3 & $15(37.5 \%)$ \\
\hline
\end{tabular}

sumab (Xgeva), duration of therapy 34 months, other risk factors: chemotherapy, diabetes, smoking; size of mucosal dehiscence $10 \times 5 \mathrm{~mm}$, area of necrotic bone $15 \times 10 \mathrm{~mm}$, recurrence after 3 months, one year after treatment, VAS 0 , stage 1 , size of dehiscence $3 \times 2 \mathrm{~mm}$.

3. Female, age 84, MRONJ in lower jaw, stage 3, VAS:7, diagnosis: myeloma, antiresorptive drug: bisphosphonate (Zometa), duration of therapy 118 months, other risk factors: chemotherapy, corticosteroids; size of mucosal dehiscence $15 \times 10 \mathrm{~mm}$, area of necrotic bone $50 \times 10 \mathrm{~mm}$, recurrence after 3 months, one year after treatment VAS 0 , stage 3 , extraoral fistula. It was not possible to remove all necrotic bone during the operation.

4. Female, age 58, MRONJ in lower jaw, stage 2 (purulent discharge), VAS:0, diagnosis: breast cancer and B lymphoma, antiresorptive drug: bisphosphonate (Zometa) and later denosumab (Xgeva), duration of therapy 45 months, other risk factors: chemotherapy, corticosteroids; size of mucosal dehiscence :multiple intraoral fistulas, area of necrotic bone 160x15 mm, recurrence after 2 months, one year after treatment VAS 0 , stage 1 , size of dehiscence $5 \times 5 \mathrm{~mm}$. It was not possible to remove all necrotic bone during the operation.

5. Male, age 85, MRONJ in upper jaw, stage 3, VAS 5, diagnosis: prostate cancer, antiresorptive drug denosumab (Xgeva), duration of therapy 24 months, other risk factor:0, size of mucosal dehiscence $35 \times 10$ $\mathrm{mm}$, area of necrotic bone $90 \times 10$, recurrence after 2 months, one year after treatment VAS 0 , stage 1 , size of dehiscence $10 \times 5 \mathrm{~mm}$. It was not possible to remove all necrotic bone during the operation.

6. Male, age 66, MRONJ in upper jaw, stage 3, VAS 5, diagnosis: prostate cancer, antiresorptive drug denosumab (Xgeva), duration of therapy 38 months, other risk factor:0, size of mucosal dehiscence $40 \times 10 \mathrm{~mm}$, area of necrotic bone $85 \times 10$, recurrence after 1 month, 
one year after treatment VAS 0 , stage 1 , size of dehiscence $25 \times 10 \mathrm{~mm}$. It was not possible to remove all necrotic bone during the operation.

From the characteristics of the six unhealed patients it is obvious that in 4 of 6 patients it was not possible to remove all necrotic bone. If we exclude these patients from final evaluation, we get a sample of 36 patients with 34 patients completely healed after one year. This is a successful outcome of surgical treatment with local PRF application in 94\%, if all necrotic bone is removed. Using Pearson's Chi-squared test with Yates' continuity correction $(P<0.05$ was considered significant) no significant effect of location, stage, diagnosis, type of antiresorptive drug or other risk factors on outcome of treatment was found. Statistical testing was limited because of the relatively small group under observation. The only statistically significant factor correlated with therapy response was size of necrotic bone surface. This was tested with Wilcoxon rank sum test with continuity correction. There was significantly higher initial defect size in patients in which complete healing was not obtained $(P=0.014)$. Size of necrotic bone was associated with therapy response Fig.4.

\section{DISCUSSION}

Though medication related osteonecrosis of jaws has become object of massive research since the first description in 2003, there is still no universally accepted therapeutic protocol. A conservative approach is usually considered as a first choice, however the results are quite disappointing ${ }^{2,5,15}$. In the study of Lesclouse et al. only $25 \%$ of necrosis healed after a conservative approach, whereas $62.5 \%$ increased in size ${ }^{4}$. Montebugnoli et al. achieved a slight reduction of necrotic area after antibiotic treatment in 7 of 9 patients but no mucosal healing. On the other hand, they had no better outcomes of treatment after surgery $^{5}$. In study of Nicolatou et al. including 67 oncological patients treated with antibiotics, healing occurred in $14.9 \%$ and pain subsided in $80.9 \%$ (ref. $^{6}$ ). Melea et al. treated 10 myeloma patients with chlorhexidine $0.12 \%$ rinses and antibiotics. Eight of these patients remained stable for a mean follow-up of 24 months (3-48), only one was completely healed after 8 months and one patient developed a higher stage of MRONJ (ref. ${ }^{16}$ ). The conservative approach often results in the improvement of symptoms or stabilisation of osteonecrosis, but it usually does not lead to complete mucosal healing. The treatment recommendations are gradually changing, and surgical therapy is now preferred and reaches better outcomes. Wutzl et al. observed 6 months after surgical treatment, complete healing with intact mucosa in $58.5 \%$ (ref. ${ }^{17}$ ). In Carlsons study of 95 resection sites 87 (91.6\%) were completely healed ${ }^{18}$. Holzinger et al. published a case series of 88 patients and overall success of surgical treatment of BRONJ was 59\% (ref. ${ }^{19}$ ). In an effort to further improve outcomes of surgical therapy various adjuvant treatment modalities have been tried. Resection of necrotic bone

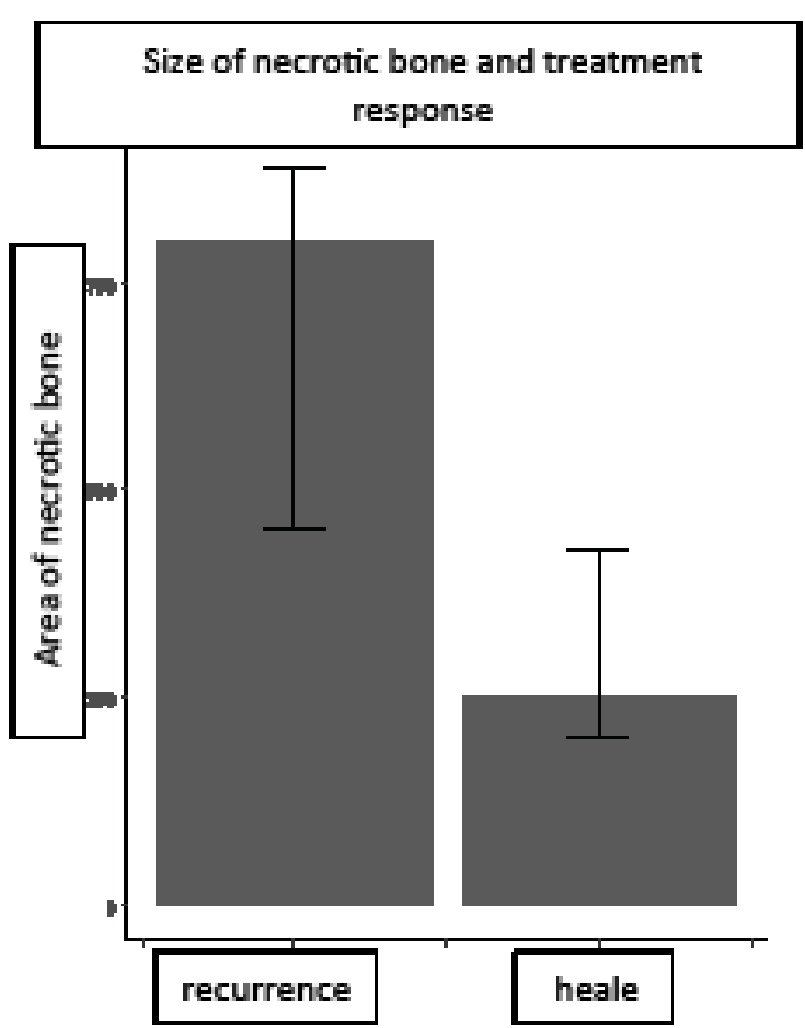

Fig. 4. Association between defect size and status of healing. Shown as median and 95\% confidence interval.

and local application of autologous platelet concentrate as adjuvant therapy, which serves as source of growth factors, shows promising results. Longo et al. reported better outcomes of surgical treatment of MRONJ with adjunctive platelet rich plasma (PRP) application than without it (94\% vs 53\%) $\left(\right.$ ref. $\left.^{20}\right)$. Bocanegra-Pérez treated 8 patients with debridement and removal of necrotic bone followed by application of PRP. After an average 14-month follow up, all patients were asymptomatic showing no exposed bone ${ }^{21}$. Adornato et al. published a 12 case series using this treatment with successful results in $83 \%$ (ref. ${ }^{22}$ ). Later, Choukroun et al. developed PRF which is called a second generation platelet concentrate. Compared to PRP, PRF is safer and has some other positive properties (prolonged release of growth factor, more stable fibrin matrix) for improving outcomes of surgical therapy ${ }^{11}$. Kim et al. in a study of 34 patients treated by resection and application of PRF reached positive outcome in $95 \%$, but only 2 patients were treated for oncological disease, the rest for osteoporosis. The follow up period was 4 months. The two unhealed patients were those with malignant disease $^{23}$. Nørholt et al. were successful with the same treatment of 15 patients ( 8 of them had malignant disease and were treated with high-dose antiresorptive medication) in $93 \%$. One patient with persistent exposed bone had renal cancer and was treated with high-dose antiresorptive medication $^{24}$. Mouro et al published a study of 11 patients with MRONJ treated with resection and PRF application and observed complete healing in $100 \%$, but in this study only patients with osteoporosis were included ${ }^{25}$. This ar- 
ticle presents a study of 40 patients with MRONJ treated with resection of necrotic bone, application of PRF and primary wound closure. Most of the patients (85\%) had malignant disease and were treated with high-dose of antiresorptive drugs. Complete healing was obtained in $85 \%$ of patients. All six unhealed patients had oncological disease and for four of them it was not possible to remove all necrotic bone during the operation, which appears to be important factor for positive outcome of surgical treatment. If these patients were excluded from evaluation, surgical therapy with resection of all necrotic bone and PRF application was successful in 94\%. No statistically significant associations among treatment response and site, stage of MRONJ, risk factors or primary disease were found, which is may be due to the small size of the group under observation. As in previous studies, all patients treated for osteoporosis did heal completely, as well as all MRONJ of zero and stage one. The only significant predictor of treatment outcome in this study was size of necrotic site, which supports early surgical interventions when treating MRONJ.

\section{CONCLUSION}

In conclusion surgical treatment of MRONJ with adjuvant local PRF application appears to be very effective, especially when all necrotic bone is removed. However, no definitive conclusion can be made due to small sample size. Even though more randomized, prospective trials are needed to confirm these outcomes, the better results of early surgical treatment compared to the conservative approach are promising.

Acknowledgement: Authors are grateful MUDr. Jaromír Gumulec Ph.D. (Department of Pathophysiology. Masaryk University) for help with statistical analysis.

Author contributions: JZ: main investigator, surgical treatment, data collection; all authors contributed in writing the manuscript and read and approved the final manuscript.

Conflict of interest statement: The authors state that there are no conflicts of interest regarding the publication of this article.

The study was approved by Institutional Ethical Committee.

\section{REFERENCES}

1. Marx RE. Pamidronate (Aredia) and zoledronate (Zometa) induced avascular necrosis of the jaws: a growing epidemic. J Oral Maxillofac Surg 2003;61(9):1115-7.

2. Ruggiero SL, Dodson TB, Fantasia J, Goodday R, Aghaloo T, Mehrotra B, O'Ryan F. American Association of Oral and Maxillofacial Surgeons position paper on medication-related osteonecrosis of the jaw 2014 update. J Oral Maxillofac Surg 2014;72(10):1938-56. doi:10.1016/j. joms.2014.04.031

3. Yoneda T, Hagino H, Sugimoto T, Ohta H, Takahashi S, Soen S, Taguchi A, Toyosawa S, Nagata T, Urade M. Bisphosphonate-related osteonecrosis of the jaw: position paper from the Allied Task Force Committee of Japanese Society for Bone and Mineral Research,
Japan Osteoporosis Society, Japanese Society of Periodontology, Japanese Society for Oral and Maxillofacial Radiology, and Japanese Society of Oral and Maxillofacial Surgeons. J Bone Miner Metab 2010;28(4):365-83. doi:10.1007/s00774-010-0162-7

4. Lesclous P, Grabar S, Abi Najm S, Carrel JP, Lombardi T, Saffar JL, Samson J. Relevance of surgical management of patients affected by bisphosphonate-associated osteonecrosis of the jaws. A prospective clinical and radiological study. Clin Oral Invest 2014;18(2):391-9. doi:10.1007/s00784-013-0979-2

5. Montebugnoli L, Felicetti L, Gissi DB, Pizzigallo A, Pelliccioni GA, Marchetti C. Biphosphonate-associated osteonecrosis can be controlled by nonsurgical management. Oral Surg Oral Med Oral Pathol Oral Radiol Endod 2007;104(4):473-7. doi:10.1016/j.tripleo.2007.01.008

6. Nicolatou-Galitis O, Papadopoulou E, Sarri T, Boziari P, Karayianni A, Kyrtsonis MC, Repousis P, Barbounis V, Migliorati CA. Osteonecrosis of the jaw in oncology patients treated with bisphosphonates: prospective experience of a dental oncology referral center. Oral Surg Oral Med Oral Pathol Oral Radiol Endod 2011;112(2):195-202. doi:10.1016/j.tripleo.2011.02.037

7. Otto S, ed. Medication-Related Osteonecrosis of the Jaws: Bisphosphonates, Denosumab, and New Agents. 2015 edition. New York: Springer; 2014.

8. Vescovi P, Merigo E, Meleti M, Manfredi M, Fornaini C, Nammour S. Surgical Approach and Laser Applications in BRONJ Osteoporotic and Cancer Patients. J Osteoporos 2012;2012. doi:10.1155/2012/585434

9. Ripamonti Cl, Cislaghi E, Mariani L, Maniezzo M. Efficacy and safety of medical ozone (O3) delivered in oil suspension applications for the treatment of osteonecrosis of the jaw in patients with bone metastases treated with bisphosphonates: Preliminary results of a phase I-II study. Oral Oncology 2011;47(3):185-90. doi:10.1016/j. oraloncology.2011.01.002

10. Freiberger JJ. Utility of Hyperbaric Oxygen in Treatment of Bisphosphonate-Related Osteonecrosis of the Jaws. J Oral Maxillofac Surg 2009;67(5,Suppl):96-106. doi:10.1016/j.joms.2008.12.003

11. Dohan DM, Choukroun J, Diss A, Dohan SL, Dohan AJ, Mouhyi J, Gogly B. Platelet-rich fibrin (PRF): a second-generation platelet concentrate. Part I: technological concepts and evolution. Oral Surg Oral Med Oral Pathol Oral Radiol Endod 2006;101(3):e37-44. doi:10.1016/j.tripleo.2005.07.008

12. Dohan DM, Choukroun J, Diss A, Dohan SL, Dohan AJ, Mouhyi J, Gogly B. Platelet-rich fibrin (PRF): a second-generation platelet concentrate. Part II: platelet-related biologic features. Oral Surg Oral Med Oral Pathol Oral Radiol Endod 2006;101(3):e45-50. doi:10.1016/j. tripleo.2005.07.009

13. Dohan DM, Choukroun J, Diss A, Dohan SL, Dohan AJ, Mouhyi J, Gogly B. Platelet-rich fibrin (PRF): a second-generation platelet concentrate. Part III: leucocyte activation: a new feature for platelet concentrates? Oral Surg Oral Med Oral Pathol Oral Radiol Endod 2006;101(3):e51-55. doi:10.1016/j.tripleo.2005.07.010

14. Choukroun J, Diss A, Simonpieri A, Girard MO, Schoeffler C, Dohan SL, Dohan AJ, Mouhyi J, Dohan DM. Platelet-rich fibrin (PRF): a second-generation platelet concentrate. Part IV: clinical effects on tissue healing. Oral Surg Oral Med Oral Pathol Oral Radiol Endod 2006;101(3):e56-60. doi:10.1016/j.tripleo.2005.07.011

15. Yoneda $T$, Hagino $H$, Sugimoto $T$, Ohta $H$, Takahashi S, Soen S, Taguchi A, Nagata T, Urade M, Shibahara T, Toyosawa S. Antiresorptive agentrelated osteonecrosis of the jaw: Position Paper 2017 of the Japanese Allied Committee on Osteonecrosis of the Jaw. J Bone Miner Metab 2017;35(1):6-19. doi:10.1007/s00774-016-0810-7

16. Melea PI, Melakopoulos I, Kastritis E, Tesseromatis Ch, Margaritis V, Dimopoulos M, Terpos E. Conservative treatment of bisphosphonate-related osteonecrosis of the jaw in multiple myeloma patients. Int J Dent 2014;2014:427273. doi:10.1155/2014/427273

17. Wutzl A, Biedermann E, Wanschitz F, Seemann R, Klug C, Baumann A, Watzinger F, Schicho K, Ewers R, Millesi G. Treatment results of bisphosphonate-related osteonecrosis of the jaws. Head Neck 2008;30(9):1224-30. doi:10.1002/hed.20864

18. Carlson ER, Basile JD. The Role of Surgical Resection in the Management of Bisphosphonate-Related Osteonecrosis of the Jaws. J Oral Maxillofac Surg 2009;67(5,Suppl):85-95. doi:10.1016/j. joms.2009.01.006

19. Holzinger D, Seemann R, Klug C, Ewers R, Millesi G, Baumann A, Wutzl A. Long-term success of surgery in bisphosphonate-related 
osteonecrosis of the jaws (BRONJs). Oral Oncology 2013;49(1):66-70. doi:10.1016/j.oraloncology.2012.07.008

20. Longo F, Guida A, Aversa C, Pavone E, Di Costanzo G, Ramaglia L, Ionna F. Platelet Rich Plasma in the Treatment of BisphosphonateRelated Osteonecrosis of the Jaw: Personal Experience and Review of the Literature. Int J Dent 2014;2014. doi:10.1155/2014/298945

21. Bocanegra-Pérez $S$, Vicente-Barrero $M$, Knezevic $M$, et al. Use of platelet-rich plasma in the treatment of bisphosphonate-related osteonecrosis of the jaw. Int. J. Oral Maxillofac. Surg 2012;41:141015. doi:10.1016/j.ijom.2012.04.020

22. Adornato MC, Morcos I, Rozanski J. The treatment of bisphosphonate-associated osteonecrosis of the jaws with bone resection and autologous platelet-derived growth factors. J. Am. Dent. Assoc 2007;138(7):971-7. doi:10.14219/jada.archive.2007.0294
23. Kim JW, Kim SJ, Kim MR. Leucocyte-rich and platelet-rich fibrin for the treatment of bisphosphonate-related osteonecrosis of the jaw: a prospective feasibility study. Brit J Oral Max Surg 2014;52(9):854-9. doi:10.1016/j.bjoms.2014.07.256

24. Nørholt SE, Hartlev J. Surgical treatment of osteonecrosis of the jaw with the use of platelet-rich fibrin: a prospective study of $15 \mathrm{pa}-$ tients. Int J Oral Maxillofac Surg 2016;45(10):1256-60. doi:10.1016/j. ijom.2016.04.010

25. Fernando de Almeida Barros Mourão C, Calasans-Maia MD, Del Fabbro M, Le Drapper Vieira F, Coutinho de Mello Machado R, Capella R, Miron RJ, Gomes Alves G. The use of Platelet-rich Fibrin in the management of medication-related osteonecrosis of the jaw: $\mathrm{A}$ case series. J Stomatol Oral Maxillofac Surg February 2020;121(1):849. doi:10.1016/j.jormas.2019.02.011 